Indian J. Pure Appl. Math., 46(4): 517-538, August 2015

(c) Indian National Science Academy

DOI: $10.1007 / \mathrm{s} 13226-015-0150-7$

\title{
FERMIONIC MEIXNER CLASSES, LIE ALGEBRAS AND QUADRATIC HAMILTONIANS
}

\author{
L. Accardi*, I. Ya. Aref'eva* and I. V. Volovich** \\ *Centro Vito Volterra, Università di Roma “Tor Vergata”, Roma I-00133, Italy \\ *Steklov Mathematical Institute, Gubkin St. 8, GSP-1, 117966, Moscow, Russia \\ e-mails:arefeva@mi.ras.ru,volovich@mi.ras.ru
}

Dedicated to Prof. Kalyan B. Sinha on occasion of his 70th birthday.

(Received 14 November 2014; after final revision 31 December 2014; accepted 12 January 2015)

\begin{abstract}
We introduce the quadratic Fermi algebra, which is a Lie algebra, and calculate the vacuum distributions of the associated Hamiltonians. In order to emphasize the difference with the Bose case, we apply a modification of the method used in the above calculation to obtain a simple and straightforward classification of the 1-dimensional Meixner laws in terms of homogeneous quadratic expressions in the Bose creation and annihilation operators. There is a huge literature of the Meixner laws but this, purely quantum probabilistic, derivation seems to be new. Finally we briefly discuss the possible multidimensional extensions of the above results.
\end{abstract}

Key words : .

\section{INTRODUCTION}

The program of quadratic quantization was introduced in the paper [7] where the realizability of this program was proved by explicit construction of the Fock representation of the renormalized quadratic Bose algebra (see Definition (3) below for the non renormalized 1-mode version). In the paper [6] the current algebra of the quadratic Bose algebra over $\mathbb{R}^{d}$ was identified with a corresponding current algebra of $s l(2, \mathbb{R})$. This allowed to include the problem of constructing representations of the renormalized quadratic Bose algebra into the general theory of factorizable representations current algebras 
of Lie algebras (see [15] and the bibliography therein) and its extension to quantum Levy processes on $*$-bi-algebras (see [8], [16]). Combining these techniques with the fact that the irreducible unitary representations of $\operatorname{sl}(2, \mathbb{R})$ are classified, in [6] a class of unitary representation of the quadratic Bose algebra was built and the vacuum distributions of the generalized field operators were identified with the 3 non standard (i.e. non Gauss or Poisson) Meixner distributions.

The problem of the existence of a Fermi analogue of the quadratic Bose algebra was investigated in [1] where:

(i) a natural candidate for the role of quadratic Fermi algebra was constructed (see Definition (1) below);

(ii) the Lie algebra isomorphism between the quadratic Fermi algebra and $\operatorname{sl}(2, \mathbb{R})$ was proved (this in particular implies the Lie algebra isomorphism between the quadratic Fermi and Bose algebras);

(iii) it was shown that the quadratic Fermi and Bose algebras cannot be $*$-isomorphic for the natural involutions induced by their explicit constructions in terms of Fermions or Bosons respectively.

The non $*$-isomorphism result, mentioned in item (iii) above, naturally rises the problem of identifying the vacuum distributions of the field operators in the Fock representation of the quadratic Fermi algebra. This problem is solved in section 2.1 of the present paper. From this one can deduce a weaker form of the above mentioned non $*$-isomorphism result, namely that there cannot exist a *-isomorphism between the two algebras mapping the Fermi quadratic annihilator into a multiple of its Bose analogue. In fact, if such a $*$-isomorphism existed, then the restriction of the Fock states of the two representations to the Cartan sub-algebras of the two algebras should give rise to the same class of probability measures. However, comparing the results of section 2.1 below with those of [6] (see also section 3 below) one immediately verifies that this is not the case.

In section 3 below it is shown that the method developed for the calculation of the Fermi vacuum distributions can be applied to the Bose case leading to a very simple and elegant classification of all possible vacuum distributions of homogeneous quadratic Boson Hamiltonians in terms of Meixner laws. On both of these topics, separately, there exists a vast literature [10], [9] for vacuum distributions of quadratic expressions of usual Boson fields (where however the connection with Meixner classes was missing) and [12], [13] for the probabilistic aspects of Meixner laws and the connection between the two was established in [6] using the theory of orthogonal polynomials. The method used here is different and we have reasons to believe that it can be extended to the multi-dimensional case (in this case the orthogonal polynomial method can only be applied to products of Meixner measures). 
The problems related with the multi-dimensional case are briefly outlined in the final section 6 .

We emphasize that the present paper is only the beginning of our investigation the quadratic Fermi algebra. In this direction there are many deep problems which have not yet an answer. Among them we mention:

(I) the existence of the Fock representation the current algebra over $\mathbb{R}^{d}$ of the quadratic Fermi algebra (for this the methods of [6] should be sufficient);

(II) in case of existence, the vacuum distribution of the corresponding fields;

(III) the validity, also in the Fermi case, of the no-go theorems proved in [17], [6], [3], [4].

These problems are now under investigation.

Finally let us mention that, after completing the present paper, we received the very interesting preprint [18] including a different approach to the notion of multi-dimensional Meixner random variables, based on the quantum decomposition of a classical multi-dimensional random variable with all moments and on its characterization in terms of commutators (see [2]). This naturally poses the problem to clarify the mutual relationships between these two approaches.

\section{The QuAdratiC Fermi Algebra: $s l_{F}(2, \mathbb{C})$ And $s l_{F, 1}(2, \mathbb{C})$}

Recall that the 1-mode CAR algebra, denoted $C A R(1)$, is the $*$-algebra with identity 1 , generators $\left\{a, a^{+}, 1\right\}$ called the Fermi creation and annihilation operators and relations

$$
\{a, a\}=\left\{a^{+}, a^{+}\right\}=0 \quad ; \quad\left\{a, a^{+}\right\}:=a a^{+}+a^{+} a=1 \quad ; \quad\left(a^{+}\right)^{*}=a
$$

As a consequence of (1), one has $a^{2}=a^{+2}=0$ which implies that both $a^{+} a$ and $a a^{+}$are orthogonal projections. Because of (1) the pair $\left\{a a^{+}, a^{+} a\right\}$ is a partition of the identity. Thus, for a 1-mode representation, the quadratic algebra $\left\{a^{2}, a^{+2}, a a^{+}\right\}=\left\{0, a a^{+}\right\}$is generated by the single projection, hence it is abelian. A possible candidate for the role of (non-trivial) quadratic Fermi algebra is constructed as follows. Consider the 2-mode CAR algebra, denoted $C A R(2)$, i.e. the associative $*$-algebra with identity $1_{F}$, generators: $a_{i}, a_{j}^{+}(i, j=1,2)$ and relations

$$
\begin{gathered}
a_{j}=\left(a_{j}^{+}\right)^{*} ;\left\{a_{i}, a_{j}\right\}=\left\{a_{i}^{+}, a_{j}^{+}\right\}=0 \quad ; \quad i, j=1,2 \\
\left\{a_{i}, a_{j}^{+}\right\}:=a_{i} a_{j}^{+}+a_{j}^{+} a_{i}=:\left[a_{i}, a_{j}^{+}\right]_{+}=\delta_{i j} \cdot 1_{F}
\end{gathered}
$$

Lemma 1 - In the above notations, defining

$$
F^{-}:=a_{1} a_{2} \quad ; \quad F^{+}:=a_{2}^{+} a_{1}^{+}=\left(F^{-}\right)^{*} \quad ; \quad N_{F}=N_{F}^{*}:=a_{2}^{+} a_{2}+a_{1}^{+} a_{1}
$$


the following quadratic Fermi commutation relations hold:

$$
\begin{array}{cc}
{\left[F^{-}, F^{+}\right]=-N_{F}+1_{F}=: M_{F} \quad ;} & {\left[N_{F}, F^{+}\right]=\left[M_{F}, F^{+}\right]=2 F^{+}} \\
{\left[N_{F}, F^{-}\right]=\left[M_{F}, F^{-}\right]=-2 F^{-}} & ;
\end{array}
$$

Remark: Notice that $N_{F} \neq 1_{F}$ therefore the right hand side of (3) is not equal to zero. Denoting

$$
S_{1}:=F^{+} \quad ; \quad S_{2}:=F^{-} \quad ; \quad S_{3}:=N_{F} \quad ; \quad S_{0}:=1_{F}
$$

(3), (4) become equivalent to

$$
\begin{aligned}
& {\left[S_{1}, S_{2}\right]=-S_{3}+S_{0} \quad ; \quad\left[S_{3}, S_{1}\right]=-2 S_{1} \quad ; \quad\left[S_{3}, S_{2}\right]=2 S_{2}} \\
& {\left[S_{0}, X\right]=0 \quad ; \quad\left(S_{1}\right)^{*}=S_{2} \quad ; \quad\left(S_{3}\right)^{*}=S_{3} \quad ; \quad\left(S_{0}\right)^{*}=S_{0}}
\end{aligned}
$$

Definition 1 - The 4-dimensional complex $*$-Lie algebra with generators $\left(S_{1}, S_{2}, S_{3}, S_{0}\right)$ and relations (5), (6) is called the quadratic Fermi algebra and denoted $s l_{F, 1}(2, \mathbb{C})$. Its 3-dimensional complex $*$-Lie sub-algebra with generators $\left(S_{1}, S_{2}, M_{F}:=-S_{3}+S_{0}\right)$ is called the Fermi $\operatorname{sl}(2, \mathbb{C})$ and denoted $s_{F}(2, \mathbb{C})$.

\subsection{Vacuum expectations of quadratic Fermi Hamiltonians}

The following problem naturally arises: Which are the vacuum distributions of the field operators of the algebra $\operatorname{sl}_{F}(2, \mathbb{C})$ ?

To discuss this problem we define, for $\alpha, \beta \in \mathbb{R}$, the homogenous quadratic Hamiltonian:

$$
H=\alpha\left(a_{1}^{+} a_{1}+a_{2}^{+} a_{2}\right)+\beta\left(a_{1}^{+} a_{2}^{+}+a_{2} a_{1}\right)
$$

and the associated time evolution, uniquely determined, on the polynomial $*$-algebra generated by the $a_{i}, a_{i}^{+}(i=1,2)$, as the solutions of the equations

$$
\partial a_{j}(t):=i\left[H, a_{j}(t)\right] \quad ; \quad a_{j}(0)=a_{j}, j=1,2
$$

Lemma 2 - Suppose $\alpha, \beta$ are arbitrary real numbers, such that

$$
\omega=\sqrt{\alpha^{2}+\beta^{2}}>0
$$

Then the Heisenberg evolutions of $a_{i}, a_{i}^{+}, i=1,2$ are given by:

$$
a_{1}^{+}(t)=\left(\cos \omega t+\frac{i \alpha}{\omega} \sin \omega t\right) a_{1}^{+}+\frac{i \beta}{\omega} \sin \omega t \cdot a_{2}
$$




$$
a_{2}^{+}(t)=\left(\cos \omega t+\frac{i \alpha}{\omega} \sin \omega t\right) a_{2}^{+}-\frac{i \beta}{\omega} \sin \omega t \cdot a_{1}
$$

ProOF : Denoting $a_{j}(t):=e^{i t H} a_{j} e^{-i t H}(j=1,2)$, the commutation relations imply that

$$
\frac{d a_{1}(t)}{d t}=-i \alpha a_{1}(t)-i \beta a_{2}^{+}(t) \quad ; \quad \frac{d a_{2}(t)}{d t}=-i \alpha a_{2}(t)+i \beta a_{1}^{+}(t)
$$

with initial condition $a_{j}^{ \pm}(0)=a_{j}^{ \pm}$. Equivalently:

$$
\frac{d}{d t}\left(\begin{array}{c}
a_{1}(t) \\
a_{2}(t) \\
a_{1}^{+}(t) \\
a_{2}^{+}(t)
\end{array}\right)=i\left(\begin{array}{cccc}
-\alpha & 0 & 0 & -\beta \\
0 & -\alpha & \beta & 0 \\
0 & \beta & \alpha & 0 \\
-\beta & 0 & 0 & \alpha
\end{array}\right)\left(\begin{array}{c}
a_{1}(t) \\
a_{2}(t) \\
a_{1}^{+}(t) \\
a_{2}^{+}(t)
\end{array}\right)=: i J\left(\begin{array}{c}
a_{1}(t) \\
a_{2}(t) \\
a_{1}^{+}(t) \\
a_{2}^{+}(t)
\end{array}\right)
$$

In the notation (13), the exponential $e^{i t J}$ is easily calculated because. $J^{2}=\left(\alpha^{2}+\beta^{2}\right) \cdot 1$ $=w^{2} \cdot 1$. Therefore

$\exp (i t J)=\left(\begin{array}{cccc}\cos \omega t-\frac{i \alpha}{\omega} \sin \omega t & 0 & 0 & -\frac{i \beta}{\omega} \sin \omega t \\ 0 & \cos \omega t-\frac{i \alpha}{\omega} \sin \omega t & \frac{i \beta}{\omega} \sin \omega t & 0 \\ 0 & \frac{i \beta}{\omega} \sin \omega t & \cos \omega t+\frac{i \alpha}{\omega} \sin \omega t & 0 \\ -\frac{i \beta}{\omega} \sin \omega t & 0 & 0 & \cos \omega t+\frac{i \alpha}{\omega} \sin \omega t\end{array}\right)$

and this is equivalent to equations (10), (11).

Lemma 3 - Denote

$$
f(t):=\left\langle\psi_{0}, e^{i t H} \psi_{0}\right\rangle
$$

Then, with $\omega$ given by (9), $f$ satisfies the equation

$$
\left(1-\frac{\beta^{2}}{\omega^{2}} \sin ^{2} \omega t\right) \frac{d f}{d t}=-\frac{\beta^{2}}{2 \omega} \sin \omega t\left(\cos \omega t+\frac{i \alpha}{\omega} \sin \omega t\right) f ; f(0)=1
$$

Proof : Let $f(t)$ be given by (14). We have

$$
\frac{d f}{d t}=i \beta\left\langle\psi_{0}, e^{i t H} a_{1}^{+} a_{2}^{+} \psi_{0}\right\rangle=i \beta\left\langle\psi_{0}, a_{2} a_{1} e^{i t H} \psi_{0}\right\rangle
$$

Using the identity:

$$
e^{i t H} a_{1}^{+} a_{2}^{+}=e^{i t H} a_{1}^{+} e^{-i t H} e^{i t H} a_{2}^{+} e^{-i t H} e^{i t H}=a_{1}^{+}(t) a_{2}^{+}(t) e^{i t H}
$$

and substituting $a_{1}^{+}(t) a_{2}^{+}(t)$ from Lemma 2, we get:

$$
\frac{d f}{d t}=i \beta\left\langle\psi_{0}, a_{1}^{+}(t) a_{2}^{+}(t) e^{i t H} \psi_{0}\right\rangle
$$




$$
=-\frac{\beta^{2}}{\omega} \sin \omega t\left(\cos \omega t+\frac{i \alpha}{\omega} \sin \omega t\right)\left\langle\psi_{0}, a_{2} a_{2}^{+} e^{i t H} \psi_{0}\right\rangle+i \beta \frac{\beta^{2}}{\omega^{2}} \sin ^{2} \omega t\left\langle\psi_{0}, a_{2} a_{1} e^{i t H} \psi_{0}\right\rangle
$$

Using (17) and

$$
\left\langle\psi_{0}, a_{2} a_{2}^{+} e^{i t H} \psi_{0}\right\rangle=\left\langle\psi_{0},\left(1-a_{2}^{+} a_{2}\right) e^{i t H} \psi_{0}\right\rangle=\left\langle\psi_{0}, e^{i t H} \psi_{0}\right\rangle=f(t)
$$

we get:

$$
\left\langle\psi_{0}, a_{2} a_{1} e^{i t H} \psi_{0}\right\rangle=\frac{1}{i \beta} \frac{d f}{d t} \Leftrightarrow \frac{d f}{d t}=-\frac{\beta^{2}}{\omega} \sin \omega t\left(\cos \omega t+\frac{i \alpha}{\omega} \sin \omega t\right) f+\frac{\beta^{2}}{\omega^{2}} \sin ^{2} \omega t \frac{d f}{d t}
$$

which is (15). The initial condition (33) is obvious.

Lemma 4 -

$$
\left\langle\psi_{0}, e^{i t H} \psi_{0}\right\rangle=\left(\cos \omega t-\frac{i \alpha}{\omega} \sin \omega t\right) e^{i \alpha t}
$$

PROOF : By direct calculations one can check that the function (18) is the solution of the differential equation (15).

Theorem 1 - The vacuum expectation $f(t)=\left\langle\psi_{0}, e^{i t H} \psi_{0}\right\rangle$ is the characteristic function of the Bernoulli random variable $X$ with distribution

$$
p_{X}(x)=\frac{1}{2}\left(\left(1-\frac{\alpha}{\omega}\right) \delta(x+\alpha+\omega)+\left(1+\frac{\alpha}{\omega}\right) \delta(x+\alpha-\omega)\right) .
$$

PROOF : Denoting $F$ the Fourier transform, from Lemma 4 one has:

$$
\begin{array}{r}
F^{-1}\left[\left(\cos \omega t-\frac{i \alpha}{\omega} \sin \omega t\right) e^{i \alpha t}\right]=\frac{1}{2} F^{-1}\left[\left(1-\frac{\alpha}{\omega}\right) e^{i(\omega+\alpha) t}+\left(1+\frac{\alpha}{\omega}\right) e^{i(\alpha-\omega) t}\right] \\
=\frac{1}{2}\left(\left(1-\frac{\alpha}{\omega}\right) \delta(x+\alpha+\omega)+\left(1+\frac{\alpha}{\omega}\right) \delta(x+\alpha-\omega)\right)
\end{array}
$$

Remark : Notice that the limit of (19) as $\omega \rightarrow 0$ in such a way that $\alpha / \omega \rightarrow c \in \mathbb{R}$ exists and (independently of $c$ ) is equal to

$$
p_{0, X}(x)=\delta(x)
$$

Definition 2 - For varying $\alpha$ and $\beta$ the family (19) can be easily seen to coincide with the class of all Bernoulli distributions. The class correspondig to $\alpha \neq \beta$ will be called the 1-st Fermionic Meixner classes. The 2-d Fermionic Meixner class, correspondig to $\alpha=\beta$, consists of the single $\delta$-measure (20). 


\section{1-MODE BOSE QUADRATIC HAMILTONIANS}

In this section we recall some known facts about quadratic 1-mode Hamiltonians. Let $\left\{\mathcal{H}, a^{+}, a, \psi_{0}\right\}$ be the (unique up to unitary equivalence) Fock representation of the 1-mode canonical commutation relations (CCR). This means that $\mathcal{H}$ is a Hilbert space, with scalar product denoted by $\langle\cdot, \cdot\rangle, \psi_{0} \in$ $\mathcal{H}$ is a unit vector, called vacuum, and $a^{+}, a$ are operators on $\mathcal{H}$, called respectively creation and annihilation operators such that, for each $n \in \mathbb{N}, \psi_{0}$ is in the domain of $\left(a^{+}\right)^{n}$, the linear span of the vectors $\left(a^{+}\right)^{n} \psi_{0}$ is dense in $\mathcal{H}$ (i. e. $\psi_{0}$ is cyclic for polynomial algebra generated by $a^{+}$and $a$ ) and on this domain the 1-mode CCR and the Fock relation

$$
\left[a, a^{+}\right]=1 \quad ; \quad a \psi_{0}=0
$$

are satisfied.

Definition 3 - The 4-dimensional complex $*$-Lie algebra with generators $\left(T_{1}, T_{2}, T_{3}, T_{0}\right)$ central element $T_{0}$ and relations

$$
\begin{gathered}
{\left[T_{1}, T_{2}\right]=T_{3}+\frac{1}{2} T_{0} \quad ; \quad\left[T_{3}, T_{1}\right]=2 T_{1} \quad ; \quad\left[T_{3}, T_{2}\right]=-2 T_{2}} \\
\left(T_{1}\right)^{*}=T_{2} \quad ; \quad\left(T_{3}\right)^{*}=T_{3} \quad ; \quad\left(T_{0}\right)^{*}=T_{0}
\end{gathered}
$$

is called the quadratic Bose algebra and denoted $s l_{B, 1}(2, \mathbb{C})$. Its 3 -dimensional complex $*$-Lie subalgebra with generators $\left(T_{1}, T_{2}, M:=T_{3}+\frac{1}{2} T_{0}\right)$ is called the Bose $s l(2, \mathbb{C})$ and denoted $s_{B}(2, \mathbb{C})$.

Remark: In the notation (21), the identifications:

$$
T_{1}:=a^{+2} / 2 \quad ; \quad T_{2}:=a^{+2} / 2 \quad ; \quad T_{3}:=a^{+} a+\frac{1}{2}
$$

give a concrete realization of the quadratic Bose algebra on the space of the Fock representation of the Heisenberg algebra. Note the difference with Schwinger bosons. Schwinger uses a doublet of bosons (see for example [19], [20]) while we use a singlet.

The most general symmetric, real homogeneous, quadratic expression in the variables $a^{+}, a$ has the form:

$$
H:=\frac{1}{2} \alpha\left(a^{+}\right)^{2}+\frac{1}{2} \bar{\alpha} a^{2}+\beta a^{+} a
$$

where $\alpha \in \mathbb{C}, \beta \in \mathbb{R} . H$ is not identically zero if and only if

$$
|\alpha|^{2}+\beta^{2}>0
$$

Without losse of generality it can be assumed that $\alpha \in \mathbb{R}_{+}$. 
In fact if $\alpha=|\alpha| e^{i \theta}$, where $\theta \in \mathbb{R}$, then the gauge transformation

$$
a \rightarrow \tilde{a}:=e^{-i \theta / 2} a \quad ; \quad a^{+} \rightarrow \tilde{a}^{+}:=e^{i \theta / 2} a^{+}
$$

leaves invariant the commutation relations (21) and the quadratic Hamiltonian $H$ in terms of $\tilde{a}$ and $\tilde{a}^{+}$becomes:

$$
H=\frac{1}{2}|\alpha|\left(\left(\tilde{a}^{+}\right)^{2}+\tilde{a}^{2}\right)+\beta \tilde{a}^{+} \tilde{a}
$$

$H$ generates the 1-parameter unitary group $U_{t}=e^{i t H}$ and the Heisenberg evolution of any operator $A$ under this group (we can simply say under $H$ ) is defined by $A(t):=e^{i t H} A e^{-i t H}$.

Lemma 5 - Let $\alpha, \beta \in \mathbb{R}$ satisfy (25) and let $\omega$ denote the positive square root of

$$
\omega^{2}:=\beta^{2}-\alpha^{2}
$$

Then, if $\omega \neq 0$ the Heisenberg evolutions of $a, a^{+}$under $H$ are given by:

$$
a^{+}(t)=\left(\cos \omega t+\frac{i \beta}{\omega} \sin \omega t\right) a^{+}+\frac{i \alpha}{\omega} \sin \omega t \cdot a
$$

If $\omega=0$, the limit of the expression (27) as $\omega \rightarrow 0$ gives:

$$
a^{+}(t)=(1+i \beta t) a^{+}+i \alpha t a
$$

Remark: Notice that the invariance of the evolutions (27), (28), under change of sign of $\omega$ implies their independence on the choice of the real square root of $\omega$.

PROOF : Notice that

$$
\frac{d a(t)}{d t}=i[H, a(t)]=-i \beta a(t)-i \alpha a^{+}(t)
$$

With the notations $a_{t} \equiv a(t), \dot{a}_{t} \equiv d a(t) / d t$, from (29) we get, for $\alpha \neq 0$

$$
i \alpha a_{t}^{+}=-i \beta a_{t}-\dot{a}_{t} \quad ; \quad a_{t}^{+}=-\frac{\beta}{\alpha} a_{t}-\frac{1}{i \alpha} \dot{a}_{t}
$$

Taking the time derivative of this equation and using (29), (30) we get

$$
-\frac{\beta}{\alpha} \dot{a}-\frac{1}{i \alpha} \ddot{a}=\dot{a}^{+}=i \beta a^{+}+i \alpha a=-\frac{i \beta^{2}}{\alpha} a-\frac{\beta}{\alpha} \dot{a}+i \alpha a
$$

Therefore in the notation (26), since $a^{ \pm}(0)=a^{ \pm}$, from (29) we get

$$
\ddot{a}+\omega^{2} a=0 \quad ; \quad \dot{a}(0)=-i \beta a-i \alpha a^{+}
$$


Thus, $a(t)$ is the solution of equation (31). Looking for a solution of the form

$$
a(t)=A \cos \omega t+B \sin \omega t
$$

we find $a=A ; \quad-i \beta a-i \alpha a^{+}=\omega B$ so that

$$
a(t)=\left(\cos \omega t-\frac{i \beta}{\omega} \sin \omega t\right) \cdot a-\frac{i \alpha}{\omega} \sin \omega t \cdot a^{+}
$$

Taking the adjoint of this, we get (27).

Finally it is clear that (28) is the limit of (27) as $\omega \rightarrow 0$ and that (28) satisfies the adjoint of equation (29) with initial condition $a^{+}(0)=a^{+}$.

Lemma 6 - Denote

$$
f(t):=\left\langle\psi_{0}, e^{i t H} \psi_{0}\right\rangle
$$

If $\omega \neq 0$ then, with $\omega$ given by (26), $f$ satisfies the equation

$$
\left(1+\frac{\alpha^{2}}{\omega^{2}} \sin ^{2} \omega t\right) \frac{d f}{d t}=-\frac{\alpha^{2}}{2 \omega} \sin \omega t\left(\cos \omega t+\frac{i \beta}{\omega} \sin \omega t\right) f ; f(0)=1
$$

Proof : Let $f(t)$ be given by (32). We have

$$
\frac{d f}{d t}=\frac{i \alpha}{2}\left\langle\psi_{0}, e^{i t H} a^{+2} \psi_{0}\right\rangle=\frac{i \alpha}{2}\left\langle\psi_{0}, a^{2} e^{i t H} \psi_{0}\right\rangle
$$

Using the identity:

$$
e^{i t H}\left(a^{+}\right)^{2}=e^{i t H} a^{+} e^{-i t H} e^{i t H} a^{+} e^{-i t H} e^{i t H}=a^{+}(t) a^{+}(t) e^{i t H}
$$

and substituting $a^{+}(t)$ from Lemma (5), we get:

$$
\begin{aligned}
\frac{d f}{d t} & =\frac{i \alpha}{2}\left\langle\psi_{0}, a^{+}(t) a^{+}(t) e^{i t H} \psi_{0}\right\rangle \\
& =-\frac{\alpha^{2}}{2 \omega} \sin \omega t\left(\cos \omega t+\frac{i \beta}{\omega} \sin \omega t\right)\left\langle\psi_{0}, a a^{+} e^{i t H} \psi_{0}\right\rangle-\frac{i \alpha^{3}}{2 \omega^{2}} \sin ^{2} \omega t\left\langle\psi_{0}, a^{2} e^{i t H} \psi_{0}\right\rangle
\end{aligned}
$$

Since

$$
\left\langle\psi_{0}, a a^{+} e^{i t H} \psi_{0}\right\rangle=\left\langle\psi_{0},\left(1+a^{+} a\right) e^{i t H} \psi_{0}\right\rangle=\left\langle\psi_{0}, e^{i t H} \psi_{0}\right\rangle=f(t)
$$

and according to (34),

$$
\left\langle\psi_{0}, a^{2} e^{i t H} \psi_{0}\right\rangle=\frac{2}{i \alpha} \frac{d f}{d t}
$$

we get:

$$
\frac{d f}{d t}=-\frac{\alpha^{2}}{2 \omega} \sin \omega t\left(\cos \omega t+\frac{i \beta}{\omega} \sin \omega t\right) f-\frac{\alpha^{2}}{\omega^{2}} \sin ^{2} \omega t \frac{d f}{d t}
$$


which is equivalent to (33). The initial condition (33) is obvious.

Lemma 7 - For $\omega^{2} \neq 0$, the vacuum expectation of the evolution operator is

$$
\begin{gathered}
f(t)=\left\langle\psi_{0}, e^{i t H(\beta, \omega)} \psi_{0}\right\rangle=\left(\frac{2 e^{-i \beta t}}{\left(1+\frac{\beta}{\omega}\right) e^{-i t \omega}+\left(1-\frac{\beta}{\omega}\right) e^{i t \omega}}\right)^{1 / 2} \\
\Leftrightarrow f(t)=\left\langle\psi_{0}, e^{i t H(\beta, \omega)} \psi_{0}\right\rangle=\left(\frac{e^{-i \beta t}}{\left(\cos \omega t-i \frac{\beta}{\omega} \sin \omega t\right)}\right)^{1 / 2}
\end{gathered}
$$

PROOF : By direct calculations one can check that the function (35) is the solution of the differential equation (33) with the initial condition (33).

Lemma 8 - If $\omega^{2}=0$, then the vacuum expectation of the evolution operator is

$$
f(t)=\left\langle\psi_{0}, e^{i t H} \psi_{0}\right\rangle=\frac{e^{-i \beta t / 2}}{(1-i \beta t)^{1 / 2}}
$$

ProOF : Repeating the proofs of Lemmata (5), (6) we find that

1. the Heisenberg evolutions of $a, a^{+}$are given by:

$$
a(t)=(1-i \beta t) a-i \beta t a^{+} \quad ; \quad a^{+}(t)=(1+i \beta t) a^{+}+i \beta t a
$$

2. $f(t)$ satisfies the following differential equation:

$$
\left(1+\beta^{2} t^{2}\right) \frac{d f}{d t}=-\frac{\beta^{2} t}{2}(i \beta t+1) f
$$

with the initial condition $f(0)=1$.

One checks directly that (36) is the solution of this equation.

Remark: Notice, the the expression (36) is the limit of the expression (35) as $\omega \rightarrow 0$.

\section{THE MEIXNER Distributions}

In this section, following [12], [13] we recall some known facts about Meixner distributions and Meixner classes. For an historical discussion on their origins we refer to [14] or to the Appendix of [5].

Recall, for completeness, that the 2-d Meixner class is the Poisson and the 1-st one is the Gaussian. 


\subsection{The 5-th Meixner class}

Definition 4 - A real valued random variable $X$ is said to have Meixner distribution with parameters $a>0, b \in(-\pi, \pi), \mu \in \mathbb{R}, \delta>0$ (or to belong to the 5 th Meixner class) if its density function is

$$
p_{X}(x ; a, b, \delta, \mu)=\frac{(2 \cos (b / 2))^{2 \delta}}{2 a \pi \Gamma(2 \delta)} \exp \left(\frac{b(x-\mu)}{a}\right)\left|\Gamma\left(\delta+\frac{i(x-\mu)}{a}\right)\right|^{2}
$$

Lemma 9 - The characteristic function of the Meixner random variable $X$ with parameters $a, b, \mu, \delta$ is

$$
E\left(e^{i t X}\right)=\left(\frac{\cos \frac{b}{2}}{\cosh \frac{a t-i b}{2}}\right)^{2 \delta} e^{i \mu t}
$$

For the proof, see [12].

\subsection{The 4-th Meixner class: Negative Binomial (Pascal) distributions}

Definition 5 - A real valued random variable $X$ is said to have Negative Binomial (or Pascal) distribution with parameters $0<p<1, r \neq 0, \mu \in \mathbb{R}$, and $d \neq 0$ (or to belong to the 4th Meixner class) if its probability density function is given by

$$
P(x ; r, p, \mu, d)=\sum_{n=0}^{\infty}\left(\begin{array}{l}
r \\
n
\end{array}\right) p^{r}(1-p)^{n} \delta(x-n d-\mu)
$$

where, by definition, for any $r \in \mathbb{R}, k \in \mathbb{N} \cup\{0\}$ :

$$
\left(\begin{array}{l}
r \\
k
\end{array}\right):=\frac{r(r+1) \ldots(r+k-1)}{k !} \quad ; \quad k \geq 1 ; \text { and }\left(\begin{array}{c}
r \\
0
\end{array}\right):=1
$$

\subsection{The 3-d Meixner class: Gamma distributions}

Definition 6 - A real valued random variable $X$ is said to have the Gamma distribution with parameters $a, \theta>0, \mu \in \mathbb{R}$ (or to belong to the 3-d Meixner class) if its density function is

$$
p_{X}(x ; a, \theta, \mu)=\frac{(x-\mu)^{a-1} e^{-(x-\mu) / \theta}}{\Gamma(a) \theta^{a}} \mathbf{1}_{[\mathbf{0}, \infty)}
$$

Lemma 10 - The characteristic function of the Gamma random variable $X$ with parameters $a, \theta, \mu$ is

$$
E\left(e^{i t X}\right)=\frac{e^{-i t \mu}}{(1-i \theta t)^{a}}
$$

Proof : This is a well known fact (for example, see [21]). 


\section{MeiXner Distributions and QuAdratic Bose Hamiltonians}

Theorem 2 - Suppose that the condition

$$
-\omega^{2}=\alpha^{2}-\beta^{2}>0
$$

is satisfied. Then the vacuum expectation $f(t)=\left\langle\psi_{0}, e^{i t H} \psi_{0}\right\rangle$, with $H$ given by (24), is the characteristic function of the Meixner type 5 random variable with parameters

$$
a=2|\omega| \quad, \quad b=2 i \log \frac{\omega+\beta}{i \alpha} \quad, \quad \mu=-\frac{\beta}{2} \quad, \quad \delta=\frac{1}{4}
$$

More explicitly:

$$
\left(\frac{2 e^{-i \beta t}}{\left(1+\frac{\beta}{\omega}\right) e^{-i t \omega}+\left(1-\frac{\beta}{\omega}\right) e^{i t \omega}}\right)^{1 / 2}=e^{i t \beta / 2}\left(\frac{\cos \frac{b}{2}}{\cosh \frac{a t-i b}{2}}\right)^{2 \cdot \frac{1}{4}}
$$

PROOF : The idea of the proof is to fit the parameters $a, b, \mu$ and $\delta$, given by (42) with those of the characteristic function given by Lemma 9. If $\omega^{2}<0$, then $\omega=-i|\omega|$. Hence,

$$
e^{a t / 2}=e^{|\omega| t}=e^{-i \omega t}
$$

Using the definition of $\omega$, we have

$$
(\omega+\beta)(\omega-\beta)=\omega^{2}-\beta^{2}=-\alpha^{2}=(i \alpha)^{2} \Leftrightarrow \frac{\omega+\beta}{i \alpha}=\frac{i \alpha}{\omega-\beta}
$$

In particular from this and (42) it follows that:

$$
e^{-i b / 2}=e^{\log \frac{\omega+\beta}{i \alpha}}=\frac{\omega+\beta}{i \alpha}=\frac{i \alpha}{\omega-\beta} \Leftrightarrow e^{i b / 2}=\frac{\omega-\beta}{i \alpha}
$$

Using (46) and recalling that $\omega$ is purely imaginary and $\beta, \alpha$ are real, we find:

$$
\cos \frac{b}{2}=R e\left(e^{-i b / 2}\right)=\frac{\omega}{i \alpha}
$$

From $(44,46)$ we have:

$$
\begin{gathered}
\cosh \frac{a t-i b}{2}=\frac{1}{2}\left(e^{a t / 2} e^{-i b / 2}+e^{-a t / 2} e^{i b / 2}\right)=\frac{1}{2}\left(e^{-i \omega t} \frac{\omega+\beta}{i \alpha}+e^{i \omega t} \frac{\omega-\beta}{i \alpha}\right) \\
=\frac{1}{2} \frac{\omega}{i \alpha}\left(\left(1+\frac{\beta}{\omega}\right) e^{-i t \omega}+\left(1-\frac{\beta}{\omega}\right) e^{i t \omega}\right)=\frac{1}{2}\left(\left(1+\frac{\beta}{\omega}\right) e^{-i t \omega}+\left(1-\frac{\beta}{\omega}\right) e^{i t \omega}\right) \cos \frac{b}{2}
\end{gathered}
$$


Replacing (47) and (48) in the characteristic function (35), we obtain:

$$
\begin{aligned}
& e^{i(\beta / 2) t}\left(\cos \frac{b}{2}\right)^{2 \cdot \frac{1}{4}}\left(\cosh \frac{a t-i b}{2}\right)^{-2 \cdot \frac{1}{4}} \\
= & e^{-i \beta t / 2}\left(\frac{\omega}{i \alpha}\right)^{-1 / 2}\left(\frac{1}{2}\right)^{-1 / 2}\left(\frac{\omega+\beta}{i \alpha} e^{-i \omega t}+\frac{\omega-\beta}{i \alpha} e^{i \omega t}\right)^{-1 / 2} \\
= & \frac{\sqrt{2} e^{-i \beta t / 2}}{\left(\left(1+\frac{\beta}{\omega}\right) e^{-i t \omega}+\left(1-\frac{\beta}{\omega}\right) e^{i t \omega}\right)^{1 / 2}}
\end{aligned}
$$

which is the vacuum expectation given by Lemma 7.

Definition 7 - We say that two characteristic functions $\phi_{1}(t)$ and $\phi_{2}(t)$ are equal up to simple transformations if $\phi_{1}(t)$ can be transformed into $\phi_{2}(t)$ by applying a finite sequence of the following transformations:

"time shift": $\phi(t) \rightarrow \phi(t) e^{i m t} \quad ; \quad m \in \mathbb{R}$

“independent copying": $\phi(t) \rightarrow \phi(t)^{q} \quad ; \quad q>0$

"time rescaling": $\phi(t) \rightarrow \phi(k t) \quad ; \quad k \neq 0$.

Proposition 1 - Up to simple transformations the characteristic function of any Meixner random variable of type 5 , can be reduced to a vacuum expectation of the form (35) for some homogeneous quadratic Bose Hamiltonian $H$ satisfying (41).

Proof : Consider the Meixner type $\mathrm{V}$ random variable $X$ with parameters $(a, b, \delta, \mu)$ and put:

$$
\alpha=\frac{a}{2}\left(\cos \frac{b}{2}\right)^{-1} \quad ; \quad \beta=\frac{a}{2} \tan \frac{b}{2}
$$

Then the vacuum expectation of the quadratic Hamiltonian, specified by these parameters, is the characteristic function $\phi_{a^{\prime}, b^{\prime}, \delta^{\prime}, \mu^{\prime}}(t)$ of Meixner distribution with parameters $a^{\prime}, b^{\prime}, \delta^{\prime}$, and $\mu^{\prime}$, where:

$$
\begin{aligned}
a^{\prime} & =2|\omega|=2\left|\sqrt{\left(\frac{a}{2} \tan \frac{b}{2}\right)^{2}-\left(\frac{a}{2}\left(\cos \frac{b}{2}\right)^{-1}\right)^{2}}\right| \\
& =a\left|\sqrt{\tan ^{2} \frac{b}{2}-\left(\cos \frac{b}{2}\right)^{-2}}\right|=a\left|\sqrt{\frac{\sin ^{2}(b / 2)-1}{\cos ^{2}(b / 2)}}\right|=a|i|=a \\
e^{\frac{-i b^{\prime}}{2}} & =\frac{\omega+\beta}{i \alpha}=\frac{\frac{a}{2} \sqrt{\tan ^{2} \frac{b}{2}-\left(\cos \frac{b}{2}\right)^{-2}}+\frac{a}{2} \tan \frac{b}{2}}{i \frac{a}{a}\left(\cos \frac{b}{2}\right)^{-1}} \\
& =\frac{i+\tan \frac{b}{2}}{i^{-1}\left(\cos \frac{b}{2}\right)^{-1}}=\cos \frac{b}{2}-i \sin \frac{b}{2}=e^{\frac{i b}{2}}
\end{aligned}
$$


and, therefore, $b^{\prime}=b, \mu^{\prime}=\frac{1}{2} b$ and $\delta^{\prime}=\frac{1}{4}$. For any $\delta, \mu$ we have:

$$
\phi_{a, b, \delta, \mu}(t)=e^{i \mu t}\left(e^{-i \mu^{\prime} t} \phi_{a, b, 1 / 4, \mu^{\prime}}(t)\right)^{4 \delta}
$$

This proves the statement.

Theorem 3 - Suppose that

$$
\operatorname{det} h=-\omega^{2}=0
$$

Then the vacuum expectation $f(t)=\left\langle\psi_{0}, e^{i t H} \psi_{0}\right\rangle$ is the characteristic function of the Gamma distribution with parameters

$$
a=1 / 2 \quad, \quad \theta=\beta \quad, \quad \mu=\beta / 2
$$

PROOF : This follows from Lemmata (8) and (10).

Remark 2: It is easy to verify that the characteristic function of any Gamma distributed random variable, up to simple transformations, is the vacuum expectation of some Bose homogeneous quadratic Hamiltonian satisfying (51).

Theorem 4 - Suppose

$$
\operatorname{det} h=-\omega^{2}<0
$$

Then the vacuum expectation $f(t)=\left\langle\psi_{0}, e^{i t H} \psi_{0}\right\rangle$ is the characteristic function of the Negative Binomial distribution with parameters

$$
r=1 / 2, d=-2 \omega, p=\left\{\begin{array}{c}
\frac{2 \omega}{\omega+\beta} \text { if } \beta>0 \\
\frac{2 \omega}{|\beta|+\omega} \text { if } \beta<0
\end{array} \quad, \quad \mu=\left\{\begin{array}{c}
\frac{\beta-\omega}{2} \text { if } \beta>0 \\
\frac{\beta-\omega}{2} \text { if } \beta<0
\end{array}\right.\right.
$$

and in both cases $p \in(0,1)$.

ProOF : From Lemma 7 we have:

$$
f(t)=\left(\frac{2 e^{-i \beta t}}{\left(1+\frac{\beta}{\omega}\right) e^{-i t \omega}+\left(1-\frac{\beta}{\omega}\right) e^{i t \omega}}\right)^{1 / 2}=e^{-i \beta t / 2}\left(\frac{\omega+\beta}{2 \omega} e^{-i \omega t}-\frac{\beta-\omega}{2 \omega} e^{i \omega t}\right)^{-1 / 2}
$$

In our case since $\omega^{2}>0$, for $p$ defined as in (53), one has

$$
1-p=\frac{\beta-\omega}{\beta+\omega}=\frac{\beta^{2}-\omega^{2}}{(\beta+\omega)^{2}}>0
$$

therefore $\beta-\omega$ and $\beta+\omega$ have the same sign. 
Case (I) : $\beta>0$. In this case

$$
p=\frac{2 \omega}{\beta+\omega}=1-\frac{\beta-\omega}{\beta+\omega} \in(0,1)
$$

and

$$
f(t)=p^{1 / 2} e^{-i \mu t}\left(1-(1-p) e^{-i d t}\right)^{-1 / 2}
$$

Using this we get the series expansion around $x=0$

$$
f(t)=K e^{-i \mu t} \sum_{n=0}^{\infty} c_{n}(1-p)^{n} e^{-i n d t}
$$

where the coefficients $c_{n}$ are given by Newton's binomial: $c_{0}=1$ and

$$
c_{n}=\left.\frac{1}{n !} \frac{d^{n}}{d x^{n}} \frac{1}{\sqrt{1-x}}\right|_{x=0}=\frac{1}{n !} \cdot \frac{1}{2} \cdot \frac{3}{2} \cdots \frac{2 n-1}{2}=\left(\begin{array}{c}
1 / 2 \\
n
\end{array}\right) \quad ; \quad n \geq 1
$$

(53) implies that $0<1-p=\frac{\beta-\omega}{\beta+\omega}<1$ and the series converges uniformly for all $t$. From this it follows that the inverse Fourier transform of $f$ is

$$
F^{-1}[f(t)](x)=\frac{1}{2 \pi} \int_{-\infty}^{\infty} e^{i x t} f(t) d t=\sum_{n=0}^{\infty}\left(\begin{array}{c}
1 / 2 \\
n
\end{array}\right) p^{-1 / 2}(1-p)^{n} \delta(x-\mu-n d)
$$

which is density function of the Pascal distribution with parameters (53).

Case (II) : $\beta<0$. In this case $0<\omega<-\beta=|\beta|$ and, in the notation (53):

$$
\begin{gathered}
f(t)=\left(\frac{2 e^{-i \beta t}}{\left(1+\frac{\beta}{\omega}\right) e^{-i t \omega}+\left(1-\frac{\beta}{\omega}\right) e^{i t \omega}}\right)^{1 / 2}=e^{-i(\omega+\beta) t / 2}\left(\frac{2 \omega}{|\beta|+\omega}\right)^{1 / 2}\left(1-\frac{|\beta|-\omega}{|\beta|+\omega} e^{-i 2 \omega t}\right)^{-1 / 2} \\
=e^{-i \mu t} p^{1 / 2}\left(1-p e^{-i d t}\right)^{-1 / 2}
\end{gathered}
$$

and the discussion is as in Case (I).

Proposition 2 - The characteristic function of any Negative Binomial distribution is, up to simple transformations, the vacuum expectation of some 1-dimensional homogeneous quadratic Bose Hamiltonian satisfying (51).

Proof : Given $0<p<1, r \neq 0, \mu \in \mathbb{R}$, and $d \neq 0$, let the parameters $\alpha$ and $\beta$ be:

$$
\alpha=\sqrt{1-\left(\frac{p}{2-p}\right)^{2}} \quad ; \quad \beta=1
$$


Then the vacuum expectation of the quadratic Bose Hamiltonian, specified by these $\alpha$ and $\beta$ is:

$$
f(t)=p^{1 / 2} e^{-i \mu^{\prime} t}\left(1-\left(1-p^{\prime}\right) e^{-i d^{\prime} t}\right)^{1 / 2}
$$

where

$$
\begin{aligned}
\omega & =\sqrt{\beta^{2}-\alpha^{2}}=\sqrt{1-\left(1-\left(\frac{p}{2-p}\right)^{2}\right)}=\frac{p}{2-p} \\
p^{\prime} & =\frac{2 \omega}{\omega+\beta}=\frac{p}{2-p}\left(\frac{p}{2-p}+1\right)^{-1}=\frac{2 p}{2-p}\left(\frac{2}{2-p}\right)^{-1}=p \\
\mu^{\prime} & =\frac{1-\omega}{\omega} \quad ; \quad d^{\prime}=-2 \omega
\end{aligned}
$$

Let us apply the following (simple) transformation:

$$
f(t) \rightarrow e^{i \mu t}\left(e^{-i \mu^{\prime} \lambda t} f(\lambda t)\right)^{2 r}
$$

where $\lambda=\frac{d}{d^{\prime}}$. We have:

$$
f(t) \rightarrow f_{2}(t)=p^{r}\left(1-(1-p) e^{i d t}\right)^{-r} e^{i \mu t}
$$

Applying again Newton's binomial formula: and repeating the computations of Theorem 4 one finds that $f_{2}(t)$ is the characteristic function of the distribution

$$
\sum_{n=0}^{\infty}\left(\begin{array}{l}
a \\
n
\end{array}\right) p^{r}(1-p)^{n} \delta(x-d n-\mu)
$$

Which is the negative Binomial distribution with parameters $p, r, \mu$, and $d$.

We summarize Remarks 1-3 in the following theorem:

Theorem 5 - The characteristic function of any Meixner type III,IV,V distribution is, up to a simple transformation, the vacuum expectation of some 1-dimensional homogeneous quadratic Bose Hamiltonian.

\section{6. $n$-DiMENSIONAL CASE}

Let $a_{i}, a_{i}^{+}, i=1,2, \ldots, n$ be Bose annihilation and creation operators, satisfying CCR:

$$
\left[a_{i}, a_{j}\right]=\left[a_{i}^{+}, a_{j}^{+}\right]=0 \quad ; \quad\left[a_{i}, a_{j}^{+}\right]=\delta_{i, j} \quad ; \quad i, j=1,2, \ldots n
$$


Because of the commutativity of the creators (annihilators), the most general Hermitean quadratic expression in the $a_{i}^{ \pm}$which is real homogeneous of degree 2 is:

$$
H_{A, C}=\sum_{i, j=1}^{n} A_{i j} a_{i}^{+} a_{j}^{+}+\sum_{i, j=1}^{n} \bar{A}_{i j} a_{i} a_{j}+\sum_{i, j=1}^{n} C_{i j} a_{i}^{+} a_{j}
$$

where $A_{i j}, C_{i j} \in \mathbb{C}$.

The Hermiteanity condition for $H_{A, C}$ and the mutual commutativity of creators (resp. annihilators) imply that one can suppose that

$$
A=A^{T} \text { (transpose) } \quad ; \quad C=C^{*}
$$

Denote the $n$-component vectors $\left(a_{i}\right)$ and $\left(a_{i}^{+}\right)$by $a$ and $a^{*}$. Then, up to an additive constant, one can rewrite the Hamiltonian (55) in matrix form:

$$
H_{A, C}=\frac{1}{2}\left(a^{*} A a^{*}+a A a\right)+a^{*} C a=\frac{1}{2}\left(a^{+}, a\right)\left(\begin{array}{cc}
C & A \\
\bar{A} & \bar{C}
\end{array}\right)=\left(\begin{array}{c}
a \\
a^{+}
\end{array}\right)=H
$$

Theorem 6 - There exist $n \times n$ matrices $\Phi(t)$ and $\Psi(t)$ such that:

$$
\left(\begin{array}{ll}
\Phi(t) & \Psi(t) \\
\bar{\Psi}(t) & \bar{\Phi}(t)
\end{array}\right)=\exp \left(i t \frac{1}{2}\left(\begin{array}{cc}
C & A \\
\bar{A} & \bar{C}
\end{array}\right)\right)
$$

Moreover, denoting $\psi_{0}$ the vacuum vector, characterized by $a_{j} \psi_{0}=0$ for all $j=1,2, \ldots n$, one has

$$
\left\langle\psi_{0}, e^{i t H_{A, C}} \psi_{0}\right\rangle=\left(\operatorname{det} \Phi(t) e^{i C t}\right)^{-1 / 2}=\operatorname{det}(\Phi(t))^{-1 / 2} \operatorname{det}\left(e^{i C t}\right)^{-1 / 2}
$$

PROOF : This result was proved by Friedrichs and extended by Berezin ([9] pg. 122).

Lemma $11-\Phi(t)$ satisfies the following equation:

$$
A^{-1} \ddot{\Phi}-i\left[C, A^{-1}\right] \dot{\Phi}+\left(C A^{-1} C-A\right) \Phi=0
$$

with the initial conditions $\Phi(0)=1, \dot{\Phi}(0)=-i C$.

ProOF : From the definition it follows that

$$
\frac{d}{d t}\left(\begin{array}{cc}
\Phi & \Psi \\
\bar{\Psi} & \bar{\Phi}
\end{array}\right)=i\left(\begin{array}{cc}
-C & -A \\
A & C
\end{array}\right)\left(\begin{array}{cc}
\Phi & \Psi \\
\bar{\Psi} & \bar{\Phi}
\end{array}\right)
$$

In particular,

$$
\dot{\Psi}=-i C \Psi-i A \bar{\Phi} \quad ; \quad \dot{\bar{\Phi}}=i A \Psi+i C \bar{\Phi}
$$


Let us express $\Psi$ using (60):

$$
\Psi=\frac{1}{i} A^{-1}(\dot{\bar{\Phi}}-i C \bar{\Phi})
$$

Substituting this for $\Psi$ into (60) we obtain:

$$
\begin{gathered}
\frac{d}{d t}\left(\frac{1}{i} A^{-1}(\dot{\bar{\Phi}}-i C \bar{\Phi})\right)=-i C\left(\frac{1}{i} A^{-1}(\dot{\bar{\Phi}}-i C \bar{\Phi})\right)-i A \bar{\Phi} \\
\quad-i A^{-1} \ddot{\bar{\Phi}}+\left(C A^{-1}-A^{-1} C\right) \dot{\bar{\Phi}}+i\left(A-C A^{-1} C\right) \bar{\Phi}=0
\end{gathered}
$$

Multiplying by $i$ and taking the adjoint, we have:

$$
A^{-1} \ddot{\Phi}+i\left(C A^{-1}-A^{-1} C\right) \dot{\Phi}+\left(C A^{-1} C-A\right) \Phi=0
$$

which is (58). The initial conditions are clear.

\subsection{Vector valued Meixner random variables}

Recall that a random variable $X$, with values in a finite dimensional real vector space $V$ (identified to its dual space) is defined by a linear map

$$
X: V \rightarrow \text { real valued random variables }
$$

An homogeneous quadratic Bose Hamiltonian $H$ of the form (55), is uniquely determined by a pair $(A, C)$ where $A$ is a symmetric complex $d \times d$ matrix and $C$ a complex self-adjoint matrix. The set of such pairs has a natural structure of finite dimensional real vector space, that we denote $V_{d}$, and the map

$$
(A, C) \mapsto H_{A, C} \in\{\text { self-adjoint operators }\}
$$

is clearly real linear. It is known that each $H_{A, C}$ can be identified to a real valued classical random variable with respect to the vacuum vector.

Definition 8 - In the above notations, a $V_{d}$-valued random variable $X$ is called of Meixner type if for each $(A, C) \in V_{d}$ the characteristic function of $X_{(A, C)}$ coincides with the vacuum characteristic function of $H_{A, C}$ :

$$
E\left(e^{i t X_{(A, C)}}\right)=\left\langle\psi_{0}, e^{i t H_{A, C}} \psi_{0}\right\rangle
$$

where $H_{A, C}$ denotes the homogeneous quadratic Fock Bose Hamiltonian with $d$-degrees of freedom, $\psi_{0}$ the corresponding vacuum vector and $E$ the expectation with respect to the random variable $X_{(A, C)}$.

Remark: From Theorem (6) we then know that Definition (8) is equivalent to require that

$$
E\left(e^{i t X_{(A, C)}}\right)=\operatorname{det}\left(\Phi(t) \cdot e^{i C t)}\right)^{-1 / 2}
$$


where $\Phi(t)$ is the solution of equation (58).

\subsection{The case of commuting $A$ and $C$}

Theorem 7 - Let A, C be $d \times d$ Hermitian complex matrices. Let $\Phi(t)$ be as in Theorem (6) and suppose that

$$
[A, C]=0
$$

and denote $\left(\alpha_{j}\right)$ (resp. $\left.\left(\beta_{j}\right)\right)$ the eigenvalues of $A$ (resp. $B$ ) and

$$
\omega_{i}^{2}:=\beta_{i}^{2}-\alpha_{i}^{2}
$$

Then

$$
\left(\operatorname{det} \Phi e^{i C t}\right)^{-1 / 2}=\prod_{j=1}^{n}\left(\frac{e^{-i t \beta_{j}}}{\cos \omega_{j} t-\frac{i \beta_{j}}{\omega_{j}} \sin \omega_{j} t}\right)^{1 / 2}
$$

Remark : In particular, under the assumptions of Theorem (7) the vacuum distribution of the symmetric operator $H_{A, C}$, given by (57) is a product of Meixner distributions.

ProOF : The identity

$$
\left(\operatorname{det} \Phi e^{i C t}\right)^{-1 / 2}=(\operatorname{det} \Phi)^{-1 / 2}\left(\operatorname{det} e^{i C t}\right)^{-1 / 2}=(\operatorname{det} \Phi)^{-1 / 2} e^{i t\left(\beta_{1}+\beta_{2}+\cdots+\beta_{n}\right)}
$$

Since $A$ and $C$ commute Eq. (58) is equivalent to

$$
\ddot{\Phi}+\left(C^{2}-A^{2}\right) \Phi=0
$$

Furthermore condition (56) implies that the *-algebra generated by $A$ and $C$ is abelian. Therefore, denoting $\phi_{i j}(i, j=1,2, \ldots, n)$ the components of $\Phi$ in the basis of eigenvector of $C$, the equation for each component are mutually independent. For the off-diagonal components we have:

$$
\frac{d^{2}}{d t^{2}} \phi_{i j}=0 \quad ; \quad \phi_{i j}(0)=0 \quad ; \quad \frac{d}{d t} \phi_{i j}(0)=0 \quad ; \quad i \neq j
$$

With the obvious solution $\phi_{i j}(t)=0$. For the diagonal components we have:

$$
\frac{d^{2}}{d t^{2}} \phi_{i i}+\omega_{i}^{2} \phi_{i i}=0 \quad ; \quad \phi_{i i}(0)=1 \quad ; \quad \dot{\phi}_{i i}(0)=-i \beta_{i}
$$

where $\omega_{i}$ is given by (64). Solving this equation, we have:

$$
\phi_{i i}(t)=\cos \omega_{i} t-\frac{i \beta_{i}}{\omega_{i}} \sin \omega_{i} t
$$

Thus $\Phi$ is a diagonal matrix and from this (65) immediately follows. 


\subsection{The 1-dimensional case}

Consider the simplest quantum model with the Hilbert space $\mathcal{H}=L^{2}(\mathbb{R})$ and the pair of creation and annihilation operators $a^{+}$and $a$ satisfying $\left[a, a^{+}\right]=1$. Denote $\psi_{0} \in \mathcal{H}$ the vector (vacuum) such that $a \psi_{0}=0$. We will determine the explicit form of the right hand side of 65 when $n=1$. In this case $A$ and $C$ are real numbers and $\Phi(t)$ is a complex valued function.

Theorem 8 - If $n=1$ then the following identity holds

$$
\left(\Phi(t) e^{i C t}\right)^{-1 / 2}=\sqrt{2} \frac{e^{-i C t / 2}}{\left[\left(1+\frac{C}{\omega}\right) e^{-i \omega t}+\left(1-\frac{C}{\omega}\right) e^{i \omega t}\right]^{1 / 2}}
$$

where

$$
\omega=\sqrt{C-|A|^{2}}
$$

PROOF : Let us verify the equality (67). From Theorem (6) one gets

$$
\frac{d}{d t}\left(\begin{array}{cc}
\dot{\Phi} & \dot{\Psi} \\
\dot{\Psi} & \dot{\Phi}
\end{array}\right)=i h\left(\begin{array}{cc}
\Phi & \Psi \\
\bar{\Psi} & \bar{\Phi}
\end{array}\right)
$$

or

$$
\dot{\Phi}=-i C \Phi-i A \bar{\Psi} \quad ; \quad \dot{\Psi}=-i C \Psi-i A \bar{\Phi}
$$

The initial boundary conditions are

$$
\Phi(0)=1, \quad \dot{\Phi}(0)=-i C ; \quad \Psi(0)=0, \quad \dot{\Psi}(0)=-i A
$$

From this one can show that $\Phi(t)$ has the form

$$
\Phi(t)=\frac{1}{2}\left[\left(1+\frac{C}{\omega}\right) e^{-i \omega t}+\left(1-\frac{C}{\omega}\right) e^{i \omega t}\right]
$$

and this proves the relation (67).

\section{ACKNOWLEDGEMENT}

This work was supported by the Russian Science Foundation N. RSF 14-11-00687, Steklov Mathematical Institute.

\section{REFERENCES}

1. L. Accardi, I. Ya. Aref'eva and I. V. Volovich, Non isomorphism of the Bose and Fermi realization of $s l(2, \mathbb{R})$. in preparation (2014). 
2. L. Accardi, H.-H. Kuo and A. Stan, Moments and commutators of probability measures, Infinite Dimensional Analysis, Quantum Probability and Related Topics, Quantum Probability Communications, 10 (2007) 591-612.

3. L. Accardi, A. Boukas and U. Franz, Renormalized powers of quantum white noise, IDA-QP (Infinite Dimensional Anal. Quantum Probab. Related Topics), 9(1) (2006), 129-147, MR2214505, DOI: 10.1142/S0219025706002263 Preprint Volterra n. 597 (2006).

4. L. Accardi and Andreas Boukas, Higher Powers of $q$-Deformed White Noise, Methods of Functional Analysis and Topology, 12(3) (2006), 205-219.

5. Luigi Accardi and Andreas Boukas, White noise calculus and stochastic calculus, in: Stochastic Analysis: Classical and Quantum, T. Hida, K. Saito (eds.) World Scientific (2005), 260-300. Proceedings International Conference on Stochastic analysis: classical and quantum, Perspectives of white noise theory, Meijo University, Nagoya, 1-5 November 2004, Preprint Volterra n. 579 (2005).

6. L. Accardi, U. Franz and M. Skeide, Renormalized squares of white noise and other non- Gaussian noises as Levy processes on real Lie algebras, Comm. Math. Phys., 228 (2002), 123-150. Preprint Volterra, N. 423 (2000).

7. L. Accardi, Y. G. Lu and I. V. Volovich, White noise approach to classical and quantum stochastic calculi, Lecture Notes of the Volterra-CIRM International School with the same title, Trento, Italy, 1999, Volterra Preprint N. 375 July (1999).

8. L. Accardi, M. Schurmann and W. von Waldenfels, Quantum independent increment processes on superalgebras, Math. ZeitSchr., 198(4) (1988), 451-477. Preprint, Heidelberg, Stochastische Mathematische Modelle, January (1987).

9. F. A. Berezin, The Method of Second Quantization, Pure Appl. Phys., 24, Academic Press, New York, 1966.

10. K. O. Friedrichs, Mathematical aspects of the quantum theory of fields, Commu Pure Appl. Math., 4 (1951), 161-224, 5(1-56) (1952), 349-411, 6 (1953), 1-72, (Collectively reissued: Interscience, New York, 1953).

11. I. S. Gradshteyn and I. M. Ryzhik, Table of Integrals, Series, and Products, Seventh Edition Hardcover by Alan Jeffrey (Editor), Daniel Zwillinger (Editor), Academic Press (2007).

12. B. Grigelionis, Generalized $z$-distributions and related stochastic processes, Lithuanian Math. J., 41 (2001), 303-319.

13. B. Grigelionis, Processes of Meixner type, Lithuanian Math. J., 39 (1999), 33-41.

14. J. Meixner, Orthogonal Polynomsysteme mit einer besonderen gestalt der erzeugenden funktion, (Orthogonal polynomial systems with a generating function of a special form), J. London Math. Soc., 9 (1934), 6-13 (Zbl. 7, 307).

15. K. R. Parthasarathy and K. Schmidt, Positive definite kernels continuous tensor products and central 
limit theorems of probability theory, Springer Lecture Notes in Mathematics no. 272 (1972).

16. M. Schürmann, White noise on bialgebras, Springer LNM 1544 (1993).

17. P. Śniady, Quadratic bosonic and free white noises, Commun. Math. Phys., 211(3) (2000), 615-628, Preprint (1999).

18. Gabriela Popa and Aurel I. Stan, Two-dimensional Meixner random vectors and their semi-quantum operators, preprint November 2014.

19. J. Schwinger, U.S. Atomic Energy Commission Report NYO-3071, 1952 or D. Mattis, The Theory of Magnetism, Harper and Row, (1982).

20. R. Anishetty, M. Mathur and I. Raychowdhury, Irreducible SU(3) Schwinger Bosons, arXiv:0901.0644.

21. Eric W. Weisstein, Gamma Distribution, From MathWorld - A Wolfram Web Resource. http://math world.wolfram.com/ GammaDistribution.html.

22. http://mathworld.wolfram.com/NegativeBinomialDistribution.html. 